\title{
Hypercomplex Algebras and Calculi Derived from Generalized Kinematics
}

\author{
Danail Brezov ${ }^{1}$ \\ ${ }^{1}$ University of Architecture Civil Engineering and Geodesy
}

June 15, 2021

\begin{abstract}
The paper provides a study of the commutative algebras generated by iteration of the cross products in $\$ \backslash$ mathbb $\{\mathrm{C}\}^{\wedge} 3 \$$. Focusing on particular real forms we also consider the analytical properties of the corresponding rings of functions and relate them to different physical problems. Familiar results from the theory of holomorphic and bi-holomorphic functions appear naturally in this context, but new types of hypercomplex calculi emerge as well. The parallel transport along smooth curves in $\$ \backslash \operatorname{mathbb}\{\mathrm{E}\}^{\wedge} 3 \$$ and the associated Maurer-Cartan form are also studied with examples from kinematics and electrodynamics. Finally, the dual extension is discussed in the context of screw calculus and Galilean mechanics; a similar construction is studied also in the multi-dimensional real and complex cases.
\end{abstract}

\section{Hosted file}

mscrip.pdf available at https://authorea.com/users/419921/articles/526309-hypercomplexalgebras-and-calculi-derived-from-generalized-kinematics

\section{Hosted file}

mscrip.tex available at https://authorea.com/users/419921/articles/526309-hypercomplexalgebras-and-calculi-derived-from-generalized-kinematics 